Please quote as: Söllner, M.; Hoffmann, A.; Hoffmann, H. \& Leimeister, J. M. (2012): How to Use Behavioral Research Insights on Trust for $\mathrm{HCl}$ System Design. In: ACM SIGCHI Conference on Human Factors in Computing Systems (CHI), Austin, Texas, USA. 


\section{How to Use Behavioral Research Insights on Trust for HCI System Design}

We present a way to systematically derive trust-supporting design elements (TSDE) using trust theory.

\section{Motivation}

- Trust has been shown to be a major antecedent of technology acceptance and usage.

- Behavioral research has created a vast amount of insights on trust building.

- Only a small fraction of the existing literature also shows ways of systematically including these insights into system design.

- Potential of most behavioral insights on trust for developing new systems often remains only partly realized.

> Behavioral research insight on trust can be systematically integrated into system design.

\section{Insights on Trust}

Trust is the belief "that an agent will help achieve an individual's goal in a situation characterized by uncertainty and vulnerability"

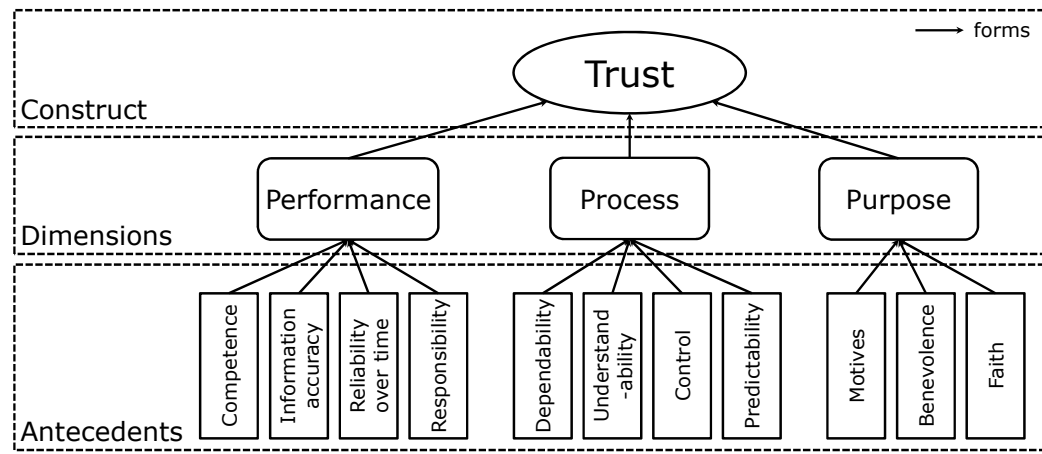

\section{Approach for Systematically Deriving TSDEs}

\begin{tabular}{|c|c|c|}
\hline $\left.\begin{array}{c}\text { Identify \& } \\
\text { prioritize } \\
\text { uncertainties }\end{array}\right\rangle \quad \begin{array}{c}\text { Select suitable } \\
\text { antecedents } \\
\text { from theory }\end{array}$
\end{tabular}
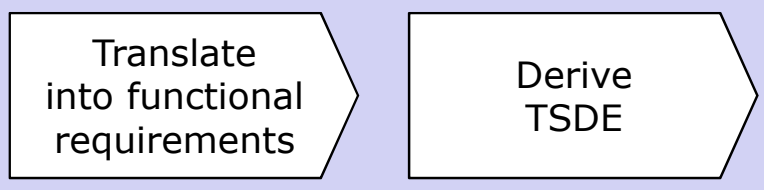

\section{Dinner Now - A Restaurant Recommendation System}

Original Version

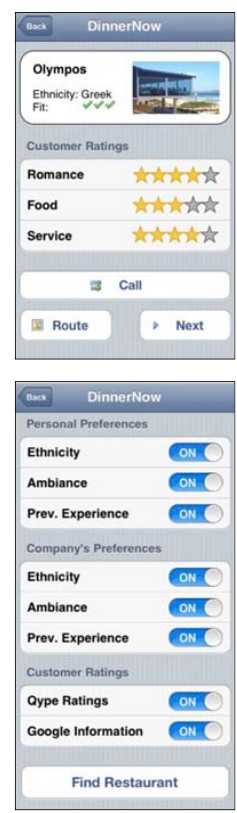

\section{Redesign}

\section{$>$ with TSDEs}
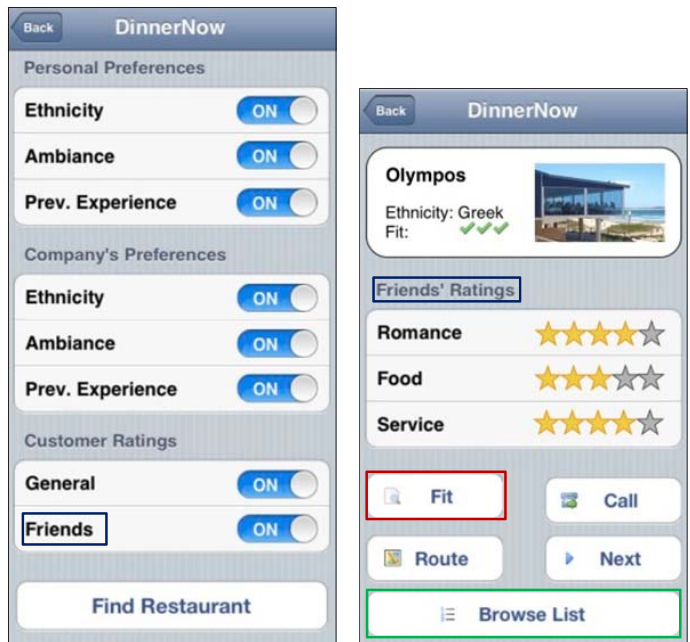

\section{Evaluation}

Using the Approach for Dinner Now

\begin{tabular}{|c|c|c|}
\hline Uncertainties & Antecedents & Functional Requirements \\
\hline $\begin{array}{l}\text { - Quality of the } \\
\text { recommendation } \\
\text { - Loss of control } \\
\text { over Dinner Now } \\
\text { - Reliability of the } \\
\text { user ratings }\end{array}$ & $\begin{array}{l}\text { - Understandability } \\
\text { - Control } \\
\text { - Information } \\
\text { accuracy }\end{array}$ & $\begin{array}{l}\text { The user should be able to } \\
\text { - receive information } \\
\text { regarding the degree the } \\
\text { configured preferences } \\
\text { were considered } \\
\text { - access the available } \\
\text { restaurants and select a } \\
\text { restaurant on his own } \\
\text { using different selection } \\
\text { criteria } \\
\text { - explicitly rely on ratings of } \\
\text { friends }\end{array}$ \\
\hline
\end{tabular}

- 166 undergraduate students were split into to groups and each group evaluated one version of the prototype.

- The TSDEs, we derived from theory using the approach, were regarded as being important by the participants.

- We observed a significant increase in the mean scores of users' trust in Dinner Now as well as their intention

to use it in the future. 


\section{How to Use Behavioral Research Insights on Trust for HCl System Design}

\author{
Matthias Söllner \\ Kassel University \\ Information Systems \\ Nora-Platiel-Str. 4 \\ Kassel, D-34127, Germany \\ soellner@uni-kassel.de \\ Axel Hoffmann \\ Kassel University \\ Information Systems \\ Nora-Platiel-Str. 4 \\ Kassel, D-34127, Germany \\ axel.hoffmann@uni-kassel.de \\ Holger Hoffmann \\ Kassel University \\ Information Systems \\ Nora-Platiel-Str. 4 \\ Kassel, D-34127, Germany \\ hoffmann@uni-kassel.de
}

\begin{abstract}
Trust has been shown to be a major antecedent of technology acceptance and usage. Consequently,

behavioral research has created vast insights on trust building. However, only a small fraction of the existing literature also shows ways of systematically including these insights into system design. Hence, the potential of most behavioral insights on trust for developing new systems often remains only partly realized. To alleviate this problem, we present a way to systematically derive trust-supporting design elements using trust theory.

Using a laboratory experiment, we show that the trustrelated design elements derived from theory are

regarded as being important by the participants, and significantly increased their trust in a restaurant recommendation system as well as in their intention to use it in the future.
\end{abstract}

\section{Author Keywords}

Theory; trust; technology acceptance and usage;

system design; laboratory experiment

\section{ACM Classification Keywords}

H.5.m [Information Interfaces and Presentation (e.g. $\mathrm{HCI})]$; 


\section{Introduction}

Even in technology-oriented fields, such as Information Systems (IS) and Human Computer Interaction (HCI), a significant number of empirical papers focus on understanding human behavior [11]. The reason is that researchers have discovered the importance of understanding human behavior for designing better systems. One heavily researched topic in this regard is "trust," as can be seen in several special issues of major journals in IS [1,2] and HCI [3]. Despite the fact that the synergetic potential of behavioral and design research has been emphasized [6], very little of the literature addresses the issue of how to use the insights created by behavioral research on trust to

systematically design more trustworthy systems.

To address this weakness, we present an approach to systematically derive trust-supporting design elements (TSDE) from theory on trust in automation [8]. We then illustrate its application to a restaurant

recommendation system and evaluate the effects of the TSDEs in a laboratory experiment, including 166 participants.

\section{The interplay between behavioral research and design research}

In general, there exist two complementary types of research: behavioral research and design research [6] Behavioral research develops and justifies theories explaining or predicting phenomena relevant for an identified need. It aims at discovering "truth." Design research builds and evaluates artifacts, which are designed to meet an identified need. It aims at creating utility. According to Hevner et al. [6], truth and utility can hardly be separated. For example, researchers may discover surprising utility in an artifact simply because a truth has not yet been discovered. On the other hand, artifacts may lack utility because a previously discovered truth was not considered when designing the artifact. In this work-in-progress paper, we focus on the latter case, arguing that several valuable insights from behavioral research on trust are not systematically considered during system design. Thus, the utility of a system is often lower than is the utility that could be achieved if behavioral research insights on trust had been systematically considered right from the beginning.

\section{Behavioral research insights on trust}

Research on technology acceptance shows that trust is a key determinant of technology adoption and usage [5]. Since the early 1990 s, a stream of HCI research has focused on trust in automation. According to Lee and See [8], automation is defined as "technology that actively selects data, transforms information, makes decisions, or controls processes" (p. 50) - a definition that fits well with most recently designed systems. Regarding trust, we adopt the definition of Lee and See [8], and define trust as the belief "that an agent will help achieve an individual's goal in a situation characterized by uncertainty and vulnerability" (p. 51).

In behavioral literature, trust is interpreted as being a multi-dimensional latent construct [7]. Consequently, research on trust in automation shares this view and identifies three dimensions forming a user's trust in automated systems: performance, process, and purpose. The performance dimension reflects the capability of the system in helping the user to achieve his goals, the process dimension reflects the user's perception regarding the degree to which the system's algorithms are appropriate, and the purpose dimension 
reflects the user's perception of the intentions that the designers of the system have.

Each of the three dimensions is formed by a number of different antecedents $[8,9]$. We focus on describing the antecedents that will be used later for deriving TSDEs for a restaurant recommendation system. A detailed description of the remaining antecedents can be found in [12]. The antecedents addressed in this work-inprogress paper are: understandability - covering the aspect of how good the user was able to understand how the system works, control - dealing with the degree to which the user has the feeling of having the system under control, and information accuracy focusing on the aspect that the information provided by the system is accurate. Figure 1 includes the dimensions and the complete set of antecedents of trust in an automated system, referring to [12].

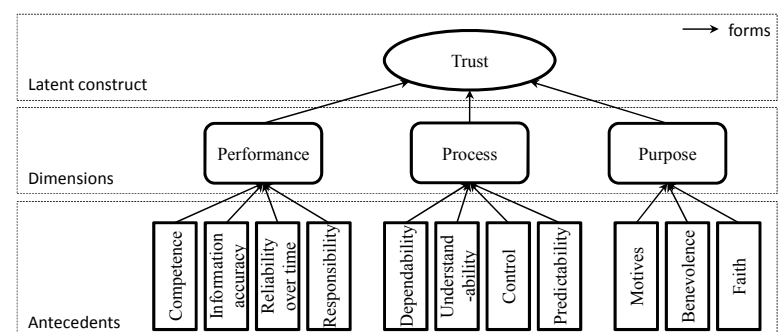

Figure 1. The formation of trust in automated systems [12].

Using behavioral research insights on trust to systematically derive TSDEs

In order to systematically derive TSDEs from theory on trust in automation, we developed an approach consisting of four steps (see Figure 2).

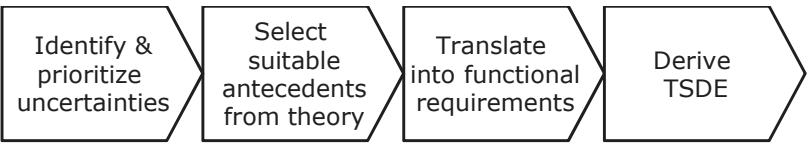

Figure 2. Approach for systematically deriving TSDEs.

As we know from the definition of trust, trust is only important in situations characterized by uncertainty. Thus, the uncertainties that the user has to face when using a particular system need to be identified first then prioritized, based on their threat to successful user adoption of the system. The prioritization is necessary, since every uncertainty that shall be countered will lead to additional development effort, and thus costs. Based on the given frame conditions (budget, time, etc.), the number of uncertainties that shall be countered need to be defined, and suitable antecedents of trust for countering these uncertainties need to be identified from theory. As is known from requirements engineering, the antecedents of a latent construct can be interpreted as under-specified functional

requirements [10]. Thus, when these requirements are considered during system design, they need to be translated into functional requirements. These functional requirements, in turn, will later be included in the software engineering approach of choice, and ultimately lead to the desired TSDEs. To further illustrate the approach, we apply the approach to derive TSDEs for a restaurant recommendation system.

\section{Dinner Now - a restaurant recommendation}

\section{system}

To show how to systematically include behavioral research insights on trust into system design, we developed an improved version of an existing prototype of a context sensitive, self-adaptive restaurant 
recommendation system, called "Dinner Now." Compared to the existing version we changed only design elements related to trust theory in order to limit the observed effects during the evaluation to the presence of the derived TSDEs.

Dinner Now allows a user to find the best restaurant for himself and his company, based upon their preferences and current location. The user's and his company's preferences regarding the ethnicity of the restaurant (style of food), the ambience, and previous experience can be included in the recommendation generation process, as well as user ratings found on the Internet. After the user has selected the data to be included and started the search, the most suitable restaurant is presented. On the restaurant screen, the user has the possibility of calling the restaurant, e.g., to request a reservation, or switching to a map that shows the shortest route from his current position to the restaurant. Alternatively, the user can generate a new recommendation if he is not satisfied with the current one.

\section{Systematically deriving TSDEs for Dinner}

Now

Following our approach, we first identified the uncertainties the user is confronted with in different situations during the interaction process with the system. For the most important uncertainties (quality of the recommendation, loss of control over Dinne Now, and reliability of the user ratings) identified by test-user prioritization, we selected one antecedent to counter each uncertainty: understandability, control, and information accuracy. This is necessary, as every requirement considered in system design increases development costs. We hence decided to reduce the number of under-specified functional requirements that would be translated into functional requirements in

order to obtain a scenario that is economically sensible.

Concretizing these antecedents resulted in the following functional requirements: Understandability - R1) after getting the recommendation, the user should be able to receive information regarding the degree to which the configured preferences were considered. Control - R2) after getting the recommendation, the user should be able to access the available restaurants and select a restaurant on his own using different selection criteria. Information accuracy - R3) the user should be able to explicitly rely on ratings of friends before a

recommendation is generated. $\mathrm{R} 4$ ) the user should be able to rely on ratings of friends for accessing the quality of a presented recommendation. The last two requirements ( $R 3$ and $R 4$ ) are based on the insight that people tend to trust their friends the most [4]. Thus,

they should perceive ratings from their friends as being more accurate than those from anonymous users. The set of four requirements were used as an input in a standard software engineering process. Figure 3 illustrates the approach for systematically deriving TSDEs using Dinner Now and the uncertainty regarding the quality of recommendation as an example. Altogether, using the approach, we were able to derive the four TSDEs highlighted in Figure 4.

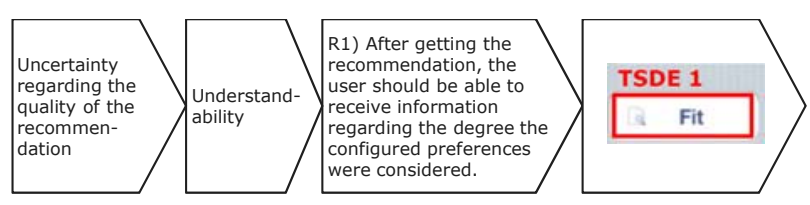

Figure 3. Example of the outcomes of each of the four steps of the approach for deriving TSDEs. 


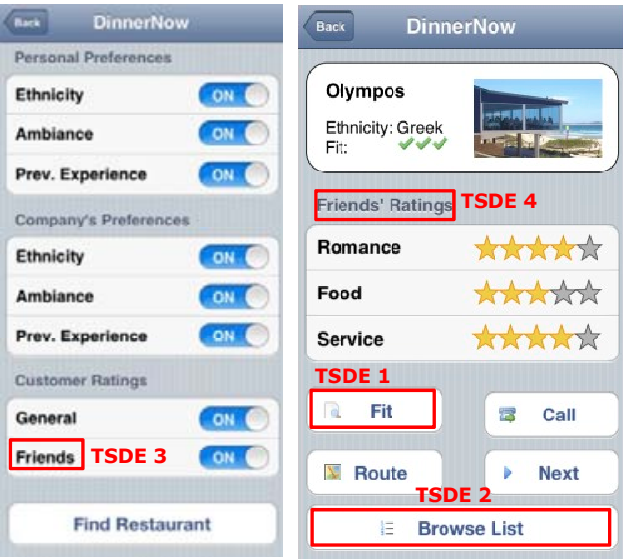

Figure 4. Two screens of "Dinner Now" including the TSDEs.

\section{Study Design}

To investigate the effects of the TSDEs implemented in Dinner Now, we recruited 166 undergraduate students (85 female, 81 male, mean age 24) to evaluate two versions (with and without the TSDEs) of the system using a between-subjects laboratory experiment. Each participant received a ten minute introduction: an

explanation of the idea and how to control the system. They were then given several tasks they had to fulfill using the system, ensuring that the participants got in touch with the full functionality of Dinner Now. This took the participants between 15 and 20 minutes. Afterwards, they were asked to fill out a questionnaire capturing the measures necessary for the evaluation (we used a bipolar 7-point Likert response format ranging from strongly disagree to strongly agree). The items were adapted from literature. After consistency checks, we included 143 questionnaires (68 referring to the system with the TSDEs) into the evaluation.

\section{Results and Discussion}

In the questionnaire, we asked participants whether they missed (version without TSDEs) or especially appreciated (version with TSDEs) the features reflecting the implemented TSDEs. The mean values that the groups reported regarding the importance of the TSDEs ranged from 5.41 to 6.01 (standard deviations ranged from 1.16 to 1.57 ). The results show that both groups regard all four TSDEs, as being important (lowest mean value is 5.41). Thus, using trust theory, we were able to derive four design elements for Dinner Now, which were regarded as being important by the participants.

The second question we intended to answer was whether this result is also reflected in the values for trust and intention to use the system in the future, as indicated by the participants. Using a t-test in SPSS 20 we are able show that the means of both the participants' trust and their intention to use the system in the future are significantly higher in the group that evaluated the system with TSDEs. The mean value for trust increased from 4.81 to 5.11 ( $p<0.075$ ), and the mean value for intention to use increased from 4.88 to $5.39(p<0.01)$.

Thus, the comparison shows that the high importance of TSDEs we deducted from theory is attested by the participants. Furthermore, the TSDEs designed to improve trust and intention to use the system in the future resulted in a significant rise of both values. The results of the evaluation show that our approach is suitable for deriving specific design elements from behavioral research insights on trust that increase users' trust in the system and lead to a higher chance of the system being adopted and used by potential users. 


\section{Conclusion and Next Steps}

The objective of this work-in-progress paper is to show that the behavioral research insight on trust can be systematically integrated into system design. We present an approach to systematically derive TSDE and apply it to redesign a restaurant recommendation system, and then evaluate the effects in a laboratory experiment. We show that our approach is feasible and the results of this first evaluation shows that the systematically derived TSDEs for the restaurant recommendation system has led to design elements that were later regarded as being important by participants, and increased their trust in the system as well as their intention to use it in the future.

Nevertheless, more research is necessary to reliably prove the value of systematically integrating behavioral research insights on trust into system design. First, although we were able to show that the approach works for one specific recommender system, we need to investigate whether the observed results hold across different recommender systems, as well as other classes of systems. Second, we evaluated the effects of the TSDEs in only one usage setting, and need to investigate whether the observed effects hold across different laboratory settings, as well as across other types of studies (e.g., field studies). Third, we evaluated the effects in only a single point in time, which was right after the participants' first usage experience. Since trust building is a dynamic process, we need to investigate whether the observed effects hold over time. Finally, the current results of the evaluation were limited to the population of undergraduate students, and thus we need to investigate whether the observed results hold across different populations.

\section{References}

11] Benbasat, I., D. Gefen, and P.A. Pavlou, Specia Issue: Trust in Online Environments. Journal of

Management Information Systems 24, 4 (2008), 5-11.

[2] Benbasat, I., D. Gefen, and P.A. Pavlou,

Introduction to the Special Issue on Novel Perspectives on Trust in Information Systems. MIS Quarterly 34, 2 (2010), 367-371.

[3] Corritore, C.L., B. Kracher, and S. Wiedenbeck, Editorial. International Journal of Human-Computer Studies 58, 6 (2003), 633-635.

[4] Forrester Research, North American

Technographics Media and Marketing Online Survey. 2009, Forrester Research, Inc.

[5] Gefen, D., E. Karahanna, and D.W. Straub, Trust and TAM in Online Shopping: An Integrated Model. MIS Quarterly 27, 1 (2003), 51-90.

[6] Hevner, A.R., S.T. March, P. Jinsoo, and S. Ram, Design Science in Information Systems Research. MIS Quarterly 28, 1 (2004), 75-105.

[7] Jarvis, C.B., S.B. Mackenzie, and P.M. Podsakoff, A Critical Review of Construct Indicators and

Measurement Model Misspecification in Marketing and

Consumer Research. Journal of Consumer Research 30 2 (2003), 199-218.

[8] Lee, J.D. and K.A. See, Trust in Automation: Designing for Appropriate Reliance. Human Factors 46, 1 (2004), 50-80.

[9] Muir, B.M., Trust in automation: Part I. Ergonomics 37, 11 (1994), $1905-1922$.

[10] Pohl, K., Requirements Engineering. 2008, Heidelberg: dpunkt. Verlag.

[11] Sidorova, A., N. Evangelopoulos, J.S. Valacich, and T. Ramakrishnan, Uncovering the Intellectual Core of the Information Systems Discipline. MIS Quarterly 32 3 (2008), 467-A20

Söllner, M., A. Hoffmann, H. Hoffmann, and J.M.

Leimeister, Towards a Theory of Explanation and

Prediction for the Formation of Trust in IT Artifacts, in SIGHCI 2011 Proceedings. Paper 6. 2011. 